\title{
EFFECTS OF ENERGY-SPECTRUM BROADENING IN ALLOYED SEMICONDUCTOR SUPERLATTICES
}

\author{
D. V. Ushakov, ${ }^{\mathrm{a}}$ V. K. Kononenko, ${ }^{\mathrm{b}}$ \\ and I. S. Manak ${ }^{\mathrm{a}}$
}

UDC 539.293:621.382

\begin{abstract}
The density of energy states is calculated for alloyed semiconductor superlattices for different excitation levels with cllowance for the effects of screening and fluctuatio's of impurity ioncentrations. The injluence of the state density tails on spontaneous-emission spectra is investigated for cross-over transitions and in a model without a selection rule for the electron wave vector. Account for the state density tails allows one to describe the longwave wing and shape of spontaneous-emission spectra in accordance with experimental data.
\end{abstract}

Key words: alloyed superlattice, tail of state density, screening, spectrum of spontaneous emission.

Alloyed superlattices (AS) with the structure of a crystal of the $n-i-p-i$-type belong to quasi-twodimensional electron systems, in which the edges of the conduction and valence bands are modulated by a periodic electrostatic potential created by charged impurities of the $n$ - and $p$-types. Depending on the degree of alloying and the pumping level of the $n-i-p-i$-crystal, the depth and shape of the potential relief change, thus affecting the properties and characteristics of such AS [1]. In the present work we consider the effects of strong alloying and give an adequate description of spontaneous-recombination spectra.

In determining the zonal parameters and optical characteristics of an AS, it is necessary to take into account the broadening of cnergy levels resulting from fluctuations of the clactrostatic potintial because of the chaotic distribution of charged impurities. Using an approach that was developed for volumetric semiconductors [2], for the distributions of the two-dimensional state density over energy $E$ in the conduction band $\rho_{c}(E)$ and the valence band $\rho_{\mathrm{v}}(E)$ we find $[3]$

$$
\begin{gathered}
\rho_{\mathrm{c}}(E)=\frac{m_{\mathrm{c}}}{2 \pi \hbar^{2} N_{\mathrm{p}}} \sum_{n} \sum_{v} \operatorname{erfc}\left(\frac{E_{\mathrm{c} 0}+E_{\mathrm{c} n v}-E}{\sigma_{\mathrm{c}}}\right)=\sum_{n} \sum_{v} \rho_{\mathrm{c} n v}(E), \\
\rho_{\mathrm{v}}(E)=\frac{m_{\mathrm{c}}}{2 \pi \hbar^{2} N_{\mathrm{p}}} \sum_{i} m_{\mathrm{v} i \perp} \sum_{n} \sum_{v} \operatorname{erfc}\left(\frac{E-E_{\mathrm{v} 0}+E_{\mathrm{vimv}}}{\sigma_{\mathrm{v}}}\right)=\sum_{i} \sum_{n} \sum_{v} \rho_{\mathrm{vimv}}(E) .
\end{gathered}
$$

\footnotetext{
*To whom correspondence should be addressed.

** Reported at the Second International Scientific and Technical Conference on Quantum Electronics, Minsk, November 23-25, 1998.

${ }^{a}$ Belarusian State University, Minsk; ${ }^{b}$ B. I. Stepanov Institute of Physics, National Academy of Sciences of Belarus, 68, F. Skorina Ave., Minsk, 220072, Belarus. Translated from Zhurnal Prikladnoi Spektroskopii, Vol. 66, No. 5, pp. 711-715, September-October, 1999. Original article submitted February 4, 1999.
} 
Here $N_{\mathrm{p}}$ is the number of AS spacings; $E_{\mathrm{c} 0}$ and $E_{\mathrm{v} 0}$ are the energies of the conduction-band bottom and the valence-band ceiling; $E_{\mathrm{c} n v}$ and $E_{\mathrm{vim} \nu}$ are the levels of dimensional quantization; $m_{\mathrm{c}}$ ar. $m_{\mathrm{v} i_{\perp}}$ are the effective mass of electrons and the transverse components of the effective masses of heavy $(i=h)$ and light $(i=1)$ holes; $\sigma_{\mathrm{c}}$ and $\sigma_{\mathrm{v}}$ are the characteristic parameters of the tails of the state density (DS) in the conduction and valence bands, respectively. We carry out the summation in Eqs. (1) and (2) over the quantum numbers of minisubbands $v$ and subbands of electrons $n$ and holes $m$. When $\sigma_{\mathrm{c}}$ and $\sigma_{\mathrm{v}} \rightarrow 0$, the formulas obtained convert to well-known expressions for a stepwise density of states [1].

In an AS, because of the spatial scattering of potential wells, screening of the fluctuating electrostatic potential, in contrast to volumetric crystals, is produced separately by electrons in the $n$-regions and holes in the $p$-regions. Therefore, the parameters of the DS tails $\sigma_{\mathrm{c}}$ and $\sigma_{\mathrm{v}}$ depend on the mean concentrations of donor $N_{\mathrm{d}}$ and acceptor $N_{\mathrm{a}}$ impurities and on the screening lengths $L_{\mathrm{c}}$ and $L_{\mathrm{v}}$ in the $n$ - and p-regions, respectively. Just as for volumetric semiconductors $[2,4,5]$, we assume that

$$
\sigma_{\mathrm{c}}=\frac{e^{2}}{\varepsilon} \sqrt{4 \pi b_{\mathrm{c}} N_{\mathrm{d}} L_{\mathrm{c}}}, \sigma_{\mathrm{v}}=\frac{e^{2}}{\varepsilon} \sqrt{4 \pi b_{\mathrm{v}} N_{\mathrm{a}} L_{\mathrm{v}}},
$$

where $\varepsilon$ is the dielectric constant of the crystal. By means of the coefficients $b_{c}$ and $b_{v}$ we take into account the quantum correction associated with the magnitude of the effective mass of current carriers and the degree of ionization of the impurities [5]. If $n_{n}$ and $p_{n}$ are the mean concintrativins of electron; and holes in an $n$-region of thickness $d_{n}$, while $n_{p}$ and $p_{p}$ are the mean concentrations of electrons and holes in a p-region of thickness $d_{p}$, then it is assumed that

$$
b_{\mathrm{c}}=1-\frac{n_{n}-p_{n}}{N_{\mathrm{d}}}, \quad b_{\mathrm{v}}=1-\frac{p_{p}-n_{p}}{N_{\mathrm{a}}} .
$$

In a linear approximation the screening can be described in a model of a two-dimensional electron gas [6] or a volumetric crystal $[4,5,7]$. Hereafter, numerical calculations for an AS on GaAs will be carried out with use of the volumetric screening length.

We find the energy levels and the potential profile of an AS by means of a self-consistent calculation of the Schrödinger and Poisson equations [1]. The values of the current-carrier concentrations are determined by the form of the envelope wave functions along the AS axis, which are normalized to the period $d=d_{n}+d_{p}+2 d_{i}$, where $d_{i}$ is the thickness of the $i$-layer. It is obvious that the quantity $n_{n}$ does not exceed $n / d_{n}$ and, correspondingly, the quantity $p_{p}$ does not exceed $p / d_{p}$, where $n$ and $p$ are the two-dimensional concentrations of electrons and holes. The change in the zonal spectrum and the effective width of the forbidden zone $E_{\mathrm{g}}^{\prime}=E_{\mathrm{c} 0}-E_{\mathrm{v} 0}$ with increase in the excitation level $r=n / N_{\mathrm{d}} d_{n}$ depends on the type of AS and its spacing $d$. Since $p=n+N$, where $N=$ $N_{\mathrm{a}} d_{p}-N_{\mathrm{d}} d_{n}$, the coefficients $b_{\mathrm{c}}$ and $b_{\mathrm{v}}$ are related to each other by the relation $b_{\mathrm{v}} \approx\left(N_{\mathrm{d}} d_{n} / N_{\mathrm{a}} d_{p}\right) b_{\mathrm{c}}$, which is strictly satisfied at $d_{i}=0$. For a compensated AS $(N=0)$ the value of $r>0$, and the quantities $b_{\mathrm{c}}$ and $b_{\mathrm{v}}$ change virtually from 1 to 0 . For an AS of the $p$-type, when $N>0$, the coefficient $b_{\mathrm{c}}>b_{\mathrm{v}}$. And, conversely, for the AS of the $n$-type $(N<0) b_{\mathrm{v}}>b_{\mathrm{c}}$.

If in addition we take into account "background" screening because of the correlation in the distribution of the impurities that appear in the course of alloying $[4,8-10]$, then we obtain

$$
L_{\mathrm{c}}=L_{\mathrm{d}}\left(1+L_{\mathrm{d}}^{2} / L_{n}^{2}\right)^{-1 / 2}, \quad L_{\mathrm{v}}=L_{\mathrm{a}}\left(1+L_{\mathrm{a}}^{2} / L_{p}^{2}\right)^{-1 / 2},
$$

where

$$
L_{n}=\left(\frac{4 \pi e^{2}}{\varepsilon}\left(\frac{d n_{n}}{d \zeta_{\mathrm{e}}}+\frac{d p_{n}}{d \zeta_{\mathrm{h}}}\right)\right)^{-1 / 2}, L_{p}=\left(\frac{4 \pi e^{2}}{\varepsilon}\left(\frac{d p_{p}}{d \zeta_{\mathrm{h}}}+\frac{d n_{p}}{d \zeta_{\mathrm{e}}}\right)\right)^{-1 / 2}
$$



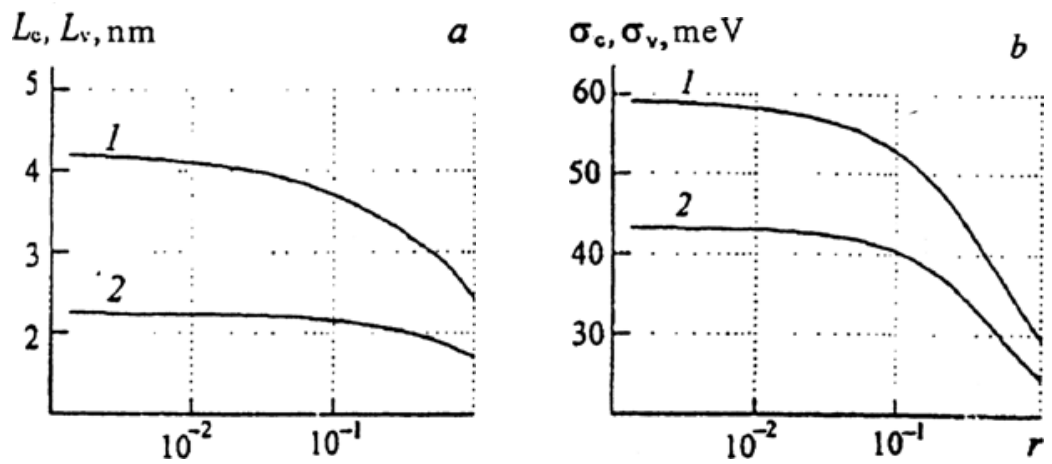

Fig. 1. Change in the screening lengths $L_{c}$ and $L_{v}$ (a) and in the DS-tail parameters $\sigma_{\mathrm{c}}$ and $\sigma_{\mathrm{v}}(\mathrm{b})$ in the $n$ - (1) and $p$-regions (2) as a function of the excitation level $r$ of a $p$-type AS; $T_{0}=1500 \mathrm{~K}, \varepsilon=12.5, E_{\mathrm{g}}=1.42 \mathrm{eV}$, crystal temperature $300 \mathrm{~K}$.

$$
L_{\mathrm{d}}=\left(\frac{4 \pi e^{2} N_{\mathrm{d}}}{\varepsilon k T_{0}}\right)^{-1 / 2}, L_{\mathrm{a}}=\left(\frac{4 \pi e^{2} N_{\mathrm{a}}}{\varepsilon k T_{0}}\right)^{-1 / 2} .
$$

Here $\zeta_{\mathrm{e}}=F_{\mathrm{e}}-E_{\mathrm{c} 0} ; \zeta_{\mathrm{h}}=E_{\mathrm{v} 0}-F_{\mathrm{h}} ; F_{\mathrm{e}}$ and $F_{\mathrm{h}}$ are the Fermi quasilevels for electrons and holes, respectively; $T_{0}$ is the "freezing" temperature of the impurities. Next, the zonal spectrum of the AS at a prescribed excitation level $r$ can be calculated by an iteration method. As the initial lengths of screening lengths in the $n$ - and $p$-regions we can take the background values of $L_{\mathrm{d}}$ and $L_{\mathrm{a}}$ and the corresponding to them values of $\sigma_{\mathrm{c}}$ and $\sigma_{\mathrm{v}}$ under the condition $b_{c}=b_{v}=1$. Then, having calculated the energy levels and the DS distribution, it is necessary to determine the screening lengths of the current carriers $L_{n}$ and $L_{p}$ and to fird from Eq. (5) the new vilues of the screening lengths in the $n$ - and $p$-regions and from Eq. (3) the characteristic parameters $\sigma_{\mathrm{c}}$ and $\sigma_{\mathrm{v}}$. This procedure is repeated until the required accuracy in determining the energy spectrum of the AS is obtained.

Results of calculations of the parameters of the energy spectrum of an AS on GaAs are presented in Fig. 1. As an example, we considered an AS of the p-type $\left(N_{\mathrm{p}}=6\right)$ with typical concentrations of impurities and thicknesses of the alloyed layers: $N_{\mathrm{a}}=8 \cdot 10^{18} \mathrm{~cm}^{-3}, N_{\mathrm{d}}=5 \cdot 10^{18} \mathrm{~cm}^{-3}, d_{\mathrm{p}}=d_{n}=15 \mathrm{~nm}$, and $d_{i}=0$. In the calculations, the values of the effective masses were taken as in the case of the crystallographic orientation of the quantum-dimensional layers of GaAs $\{100\}: m_{\mathrm{c}}=0.067 m_{\mathrm{e}}, m_{\mathrm{vh}}=0.34 m_{\mathrm{e}}, m_{\mathrm{vl}}=0.094 m_{\mathrm{e}}, m_{\mathrm{mh} \perp}=0.11 m_{\mathrm{e}}, m_{\mathrm{v} \perp \perp}=$ $0.20 m_{\mathrm{e}}$. Here $m_{\mathrm{vh}}$ and $m_{\mathrm{vl}}$ are the longitudinal components of the effective masses of the holes, which specify the spectrum of levels of dimensional quantization in the potential wells.

As is seen from Fig. 1, with an increase in the excitation level of the AS the screening lengths in the $n$ and $p$-regions decrease noticeably, especially in the $n$-region, and, correspondingly, the DS tails are reduced. At small pumpings $(r<0.1)$ the DS tail in the $n$-region is mainly determined by the background screening, while in the p-region the correlation effect in the distribution of the impurities is weakened because of screening of the impurity potential by equilibrium holes. The background screening is specified by the mean concentrations of alloying impurities and the characteristic temperature $T_{0}$. The quantity $T_{0}$ can be taken close to the melting point of the crystal $(1500 \mathrm{~K})[8,9]$. In growing the AS, the substrate tempirature is usu: $1 \mathrm{l} y$ much lower and amounts to $-820 \mathrm{~K}[11]$. However, as numerical evaluations show, a change in the quantity $1_{\hat{u}}$ within these limits has no effect on the final calculations of the DS tails and the emission spectra.

We calculated the radiative-recombination rate both in the case of cross-over transitions (CT) [12] and in a model without a selection rule (WSR) for the electron wave vector $[1,13]$. In the model of CT with allowance for the DS tails the spontaneous-radiation spectrum is described by the expression [14] 

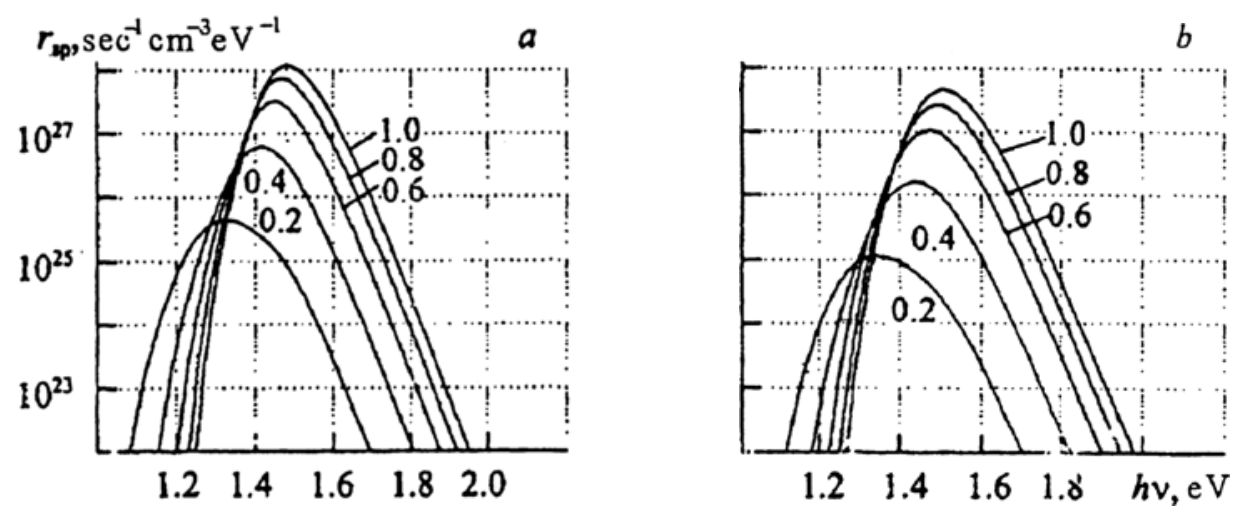

Fig. 2. Spontaneous-recombination rate $r_{\mathrm{sp}}(h v)$ for CT (a) and in the WSR model (b) for different values of the pumping parameter $r$ (the numbers at the curves).

$$
r_{\mathrm{sp}}(h v)=\frac{A_{\mathrm{cv}}}{2 \pi \hbar^{2} N_{\mathrm{p}} d} \sum_{i} m_{r i \perp} \sum_{n} \sum_{m} \sum_{v} \operatorname{erfc}\left(\frac{h v-h v_{n m i v}}{\sigma_{\mathrm{cv}}}\right)\left|I_{n m i v}\right|^{2} f_{\mathrm{e}}\left(E_{\mathrm{c} n m i v}\right) f_{\mathrm{h}}\left(E_{\mathrm{v} n m i v}\right),
$$

where $A_{\mathrm{cv}}$ is the Einstein coefficient; $m_{\mathrm{ril}}$ is the reduced mass; $h v_{n m i v}=E_{\mathrm{g}}^{\prime}+E_{\mathrm{c} n \nu}+E_{\mathrm{vimv}} ; \sigma_{\mathrm{cv}}=\sqrt{\sigma_{\mathrm{c}}^{2}+\sigma_{\mathrm{v}}^{2}} ; I_{n m i v}$ is the overlapp integral of the envelope wave functions for electrons and holes; $f_{\mathrm{e}}\left(E_{\mathrm{cnmi \nu}}\right)$ and $f_{\mathrm{h}}\left(E_{\mathrm{vnmi \nu}}\right)$ are the Fermi-Dirac distribution functions for electrons and holes with energies that are determined as

$$
\begin{gathered}
E_{\mathrm{c} n m i v}=E_{\mathrm{c} 0}+\frac{m_{\mathrm{r} i \perp}}{m_{\mathrm{c}}}\left(h v-E_{\mathrm{g}}^{\prime}\right)+\frac{m_{\mathrm{r} i \perp}}{m_{\mathrm{v} i \perp}} E_{\mathrm{c} n v}-\frac{m_{\mathrm{r} i \perp}}{m_{\mathrm{c}}} E_{\mathrm{v} i m v} \\
E_{\mathrm{v} n m i v}=E_{\mathrm{v} 0}+\frac{m_{\mathrm{r} i \perp}}{m_{\mathrm{v} i \perp}}\left(h v-E_{\mathrm{g}}^{\prime}\right)+\frac{m_{\mathrm{r} i \perp}}{m_{\mathrm{v} i \perp}} E_{\mathrm{c} n v}-\frac{m_{\mathrm{r} i \perp}}{m_{\mathrm{c}}} E_{\mathrm{v} i m v} .
\end{gathered}
$$

Here it is assumed that the energy fluctuations of the edges of the conduction and valence bands in the $n$ - and $p$-regions of the AS are independent and that for the energy levels $E_{\mathrm{cnmiv}}$ and $E_{\mathrm{vnmiv}}$ in the bands between which optical transitions occur, the mean values are taken, as in the case of the fluctuation mechanism of broadening of spectral lines in quantum-dimensional heterolasers [15]. The dependence of the forbidden-zone width of the semiconductor $E_{\mathrm{g}}$ on the temperature $T$ is also taken into account.

In the case of optical transitions WSR for the electron wave vector, for the spontaneous-recombination rate we obtain

$$
r_{\mathrm{sp}}(h v)=\frac{A}{d^{2}} \sum_{i} \sum_{n} \sum_{m} \sum_{v} \int \rho_{\mathrm{cn} v}(E) \rho_{\mathrm{vim} v^{\prime}}(E-h v)\left|I_{n m i v}\right|^{2} f_{\mathrm{e}}(E) f_{\mathrm{h}}(E-h v) d E,
$$

where $A=32 \pi a_{0}^{3} A_{\mathrm{cv}}$ is the probability of optical transitions WSR; $a_{0}$ is the Bohr effective radius of the impurity; the DS in the minisubbands $\rho_{\mathrm{cn} v}$ and $\rho_{\mathrm{vim} v}$ correspond to Eqs. (1) and (2). For the probability of optical transitions we take the value for volumetric strongly alloyed semiconductors with $\tau_{0}=1.7 \mathrm{~nm}$, an:l we ignore ihe dependence of $A$ on the dimensions of the quantum wells [16], which can be substantial in the case of short-spacing AS.

Figure 2 presents calculated spontaneous-emission spectra of an AS with parameters identical to those given in Fig. 1. The intensity and decay of the emission spectrum on the short-wave wing are determined by the Fermi distribution of nonequilibrium current carriers, i.e., by the excitation level and the crystal temperature. The longwave wing of the spectra depends on the distribution of the DS on the DS tails, and it also changes with the crystal pumping. An increase in $r$ leads to a reduction of the DS tails and, correspondingly, growth of the slope of 

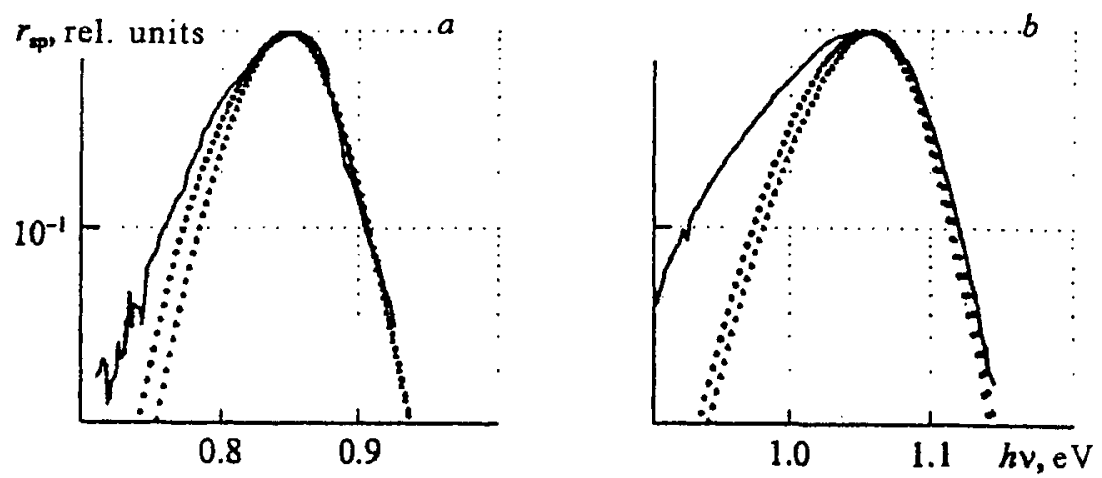

Fig. 3. Spontaneous-emission spectra of an AS on GaAs calculated for different excitation levels: $\Delta F=0.85 \mathrm{eV}, r=0.001$ (a); $\Delta F=1.08 \mathrm{eV}, r=0.1$ (b). $N_{\mathrm{a}}=10^{19} \mathrm{~cm}^{-3}, N_{\mathrm{d}}=4 \cdot 10^{18} \mathrm{~cm}^{-3}, d_{p}=35 \mathrm{~nm}, d_{n}=25 \mathrm{~nm}, d_{i}=0, T$ $=90 \mathrm{~K}, E_{\mathrm{g}}=1.50 \mathrm{eV}$. The solid line denotes experimental data of [7];o) the case of $\mathrm{CT} ; \Delta)$ the WSR model.

the dependence of the intensity $r_{\mathrm{sp}}$ on the light frequency $\nu$ on the longwave wing of the spectra. Here the emissionband width changes only slightly with $r$. A comparison between Fig. 2a and Fig. $2 b$ shows that calculations for various mechanisms of radiative recombination lead to virtually the same results.

In order to establish the applicability of the approach developed for describing spontaneous-radiation spectra of an AS, we will analyze the experimental data of $[7,17]$. Photoluminescence spectra of an AS on GaAs measured at $T=90 \mathrm{~K}$ reveal a wide low-energy tail and the absence of a structure associated with dimensional quantization [7]. The theoretical spectra for the two mechanisms of recombination have a similar smooth shape (see Fig. 3). The excitation level of the analyzed structure $\Delta F=F_{\mathrm{e}}-F_{\mathrm{h}}$ was selected in such a way that the high-energy edge of the calculated spectrum would coincide with that measured experimentally in relative units. In contrast to the modified model of exponential DS tails suggested in [7], the approach developed in this work describes well the spontaneous-radiation spectrum with weak excitation. As the excitation of the AS increases to $r \approx 0.1$ and above, the longwave edge of the spectrum is not reduced in practice and is even expanded slightly. This discrepancy between the experimental and theoretical spectra can apparently be eliminated by means of a more detailed analysis of the nature of the screening of the impurity potential and the degree of ionization of the impurities and by allowance for the relaxational mechanism of broadening of the spectral lines.

Results of calculations of radiation spectra of a $\delta$-AS on GaAs are presented in Fig. 4. As can be seen, the DS tails smooth the spontaneous-radiation spectra and specify the longwave wing. The spectra calculated agree well with the experimental data of [17] both in the case of weak excitation and at a considerable level of pumping by an electric current. Thus, the approach developed with account for Gaussian fluctuations of the impurity potential describes adequately the luminescence spectra of the $\delta$-AS. The calculations of the spectra carried out by the method suggested are less laborious compared to the model of [17], where the authors used an asymmetric probability density distribution function of a fluctuating potential in relation to the courdinate aiong the AS axis.

From the analysis performed it is evident that a description of the optical spectra of an AS requires consideration of fluctuations in the alloying-impurity concentrations and the screening process with change in the excitation level. The longwave wing of the emission spectra is determined mainly by the distributions in the DS tails in the conduction and valence bands. The levels of dimensional quantization are not clearly manifested in the form of the curves of the emission spectra, which are virtually independent of the selection rule. The procedure developed for calculating the rate of optical transitions allows one to describe not only the photo- and electroluminescence spectra of AS but also the change in their spectra of amplification and absorption with the pumping. 

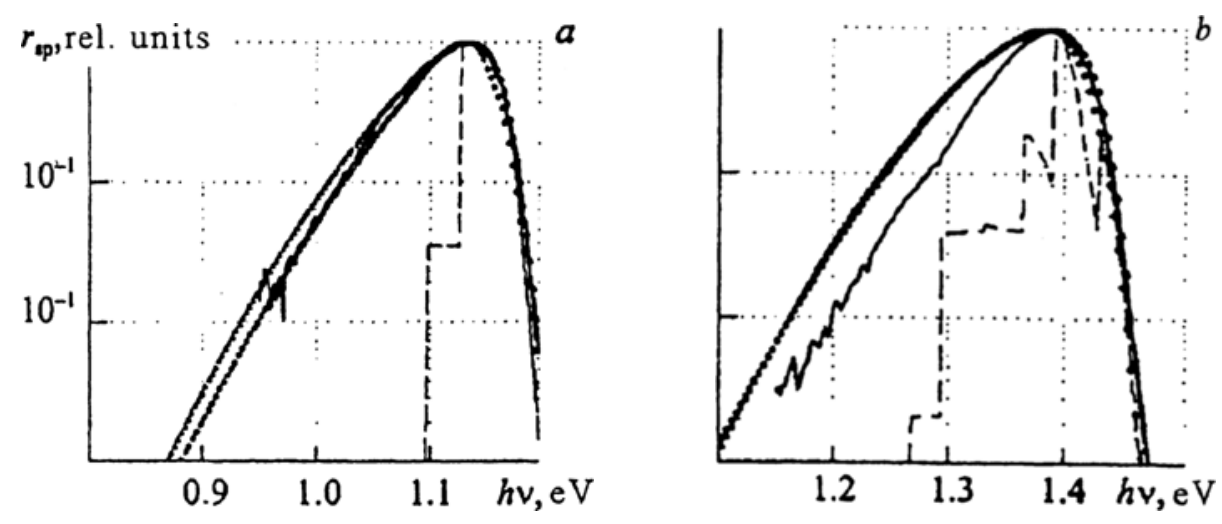

Fig. 4. Spontaneous-emission spectra of a $\delta$-AS on GaAs calculated for different excitation levels: $\Delta F=1.17 \mathrm{eV}, r=0.25$ (a); $\Delta F=1.43 \mathrm{eV}, r=0.76$ (b). $N_{\mathrm{a}}=8 \cdot 10^{19} \mathrm{~cm}^{-3}, N_{\mathrm{d}}=6 \cdot 10^{19} \mathrm{~cm}^{-3}, d_{p}=d_{n}=1 \mathrm{~nm}, d_{i}=14 \mathrm{~nm}, T=$ $50 \mathrm{~K}, E_{\mathrm{g}}=1.51 \mathrm{eV}$. The solid line denotes experimental data of [17]; the dashed line, calculation without allowance for DS tails; o) the case of CT; $\Delta$ ) the WSR model.

\section{REFERENCES}

1. V. K. Kononenko, I. S. Manak, and D. V. Ushakov, Proc. SPIE, 3580, 10-27 (1998).

2. E. O. Kane, Phys. Rev., 131, 79-88 (1963).

3. D. V. Ushakov, V. K. Kononenko, and I. S. Manak, in: Proc. IIIrd Conf. on Laser Physics and Spectroscopy, Grodno, 1997, Vol. 1, Minsk (1997), pp. 384-386.

4. B. I. Shklovskii and A. L. Éfros, Electron Properties of Alloyed Semiconductors [in Russian ], Moscow (1979).

5. V. K. Kononenko, Charge Transfer and Screening in Strongly Alloyed Semiconductors, Preprint No. 224 of the Institute of Physics, Academy of Sciences of the BSSR [in Russian ], Minsk (1980).

6. A. Ya. Shik, Fiz. Tekh Poluprovodn., 29, 1345-1381 (1995).

7. M. Renn, C. Metzner, and G. H. Döhler, Phys. Rev. B, 48, 11220-11227 (1993).

8. L. V. Keldysh and G. P. Proshko, Fiz. Tverd. Tela, 5, 3378-3389 (1963).

9. F. Stern, Phys. Rev. B, 3, 3559-3560 (1971).

10. V. K. Kononenko, Zh. Prikl. Spekstrosk., 41, 106-110 (1984).

11. K. Plog, in: L. Cheng and K. Plog (eds.), Molecular-Beam Epitaxy and Heterostructures [Russian translation ], Moscow (1989), pp. 428-461.

12. D. V. Ushakov and V. K. Kononenko, in: Physics, Chemistry, and Application of Nanostructures, Singapore (1997), pp. 121-124.

13. D. V. Ushakov and V. K. Kononenko, Proc. SPIE, 3423, 120-123 (1998).

14. D. V. Ushakov, V. K. Kononenko, and I. S. Manak, Quantum Electronics. Proc. 2nd Int. Scientific and Technical Conf., Minsk (1998), pp. 20-21.

15. D. V. Karasev and V. K. Kononenko, Zh. Prikl. Spektrosk., 61, 404-408 (1994).

16. A. A. Afonenko, V. K. Kononenko, I. S. Manak, and V. A. Shevtsov, Fiz. Tekh. Poluprovodn., 31, 1087-1091 (1997).

17. C. Metzner, K. Schrüfer, U. Wieser, M. Luber, M. Kneissl, and G. H. Döhler, Phys. Rev. B, 51, 5106-5115 (1995). 